\title{
Evaluating the provision of service for percutaneous endoscopic gastrostomy (PEG) within the United Kingdom (UK): results of a national survey
}

\author{
M. Kurien ${ }^{1}$, C. Romaya ${ }^{2}$ and D. S. Sanders ${ }^{1}$ \\ ${ }^{1}$ Department of Gastroenterology, Royal Hallamshire Hospital, Sheffield and ${ }^{2}$ British Society of Gastroenterology, \\ London, $U K$
}

Controversies exist about PEG feeding because of the significant morbidity and mortality associated with the procedure and the lack of evidence regarding its use in certain indications. We aimed to establish the provision of service for PEG that currently exists within the UK. All UK NHS hospitals providing an endoscopy service $(n=260)$ were invited to participate in this study. A custom designed web based questionnaire was sent to all providers enquiring about service arrangements for PEG. Questions were asked regarding the number of PEG insertions undertaken, referral practice, specialists performing the procedure, the time interval between referral and PEG insertion, triage systems, PEG aftercare, local guidelines and prophylactic antibiotic usage.

Questionnaires were returned by 215 of 260 hospitals (82.7\%), of which 57.2\% (123/215) were Joint Advisory Group (JAG) accredited units. Specialists inserting PEGs within these hospitals included Gastroenterologists (100\%), Surgeons (33\%), Nurse Endoscopists (36\%), Specialist Registrars $(51 \%)$ and Geriatricians (3\%). More than 50 PEGs per year were inserted by 32.5\% (70/215) of hospitals, with 4 hospitals inserting more than 150. $18 \%$ (38/215) of units were low-volume service providers, undertaking fewer than 25 procedures a year. Stroke and neurodegenerative conditions were the main indications for PEG insertion, with 36\% (77/215) of hospitals inserting PEGs for dementia.

There were variations in the timing of PEG insertion, with $33 \%(72 / 215)$ having a strict policy of waiting more than 2 weeks from referral to insertion, $14 \%$ (30/215) performing immediately and $34 \%(74 / 215)$ determining the time delay dependent upon the underlying condition. Local guidelines for PEG insertion existed in $87 \%(186 / 215)$ of hospitals and 78\% (168/215) had access to radiologically inserted gastrostomies. Prophylactic antibiotics were used in $93 \%(201 / 215)$ of hospitals'. Only 64\% (137/215) had a dedicated PEG aftercare service.

There were no differences when comparing; JAG (34\%) and non-JAG centres (38\%) for PEG insertion in dementia p $=0.55$ and JAG $(96 \%)$ and non-JAG centres $(90 \%)$ for antibiotic use $(\mathrm{p}=0.09)$. When comparing low volume centres $(<25)$ to high volume centres $(>150)$ no differences in practice could be observed (example, dementia, $\mathrm{p}=0.38$ ).

This is the most comprehensive study evaluating provision of service for PEG within the UK. We have described variations in practice (particularly with regards to the policy for dementia and number of procedures undertaken). This may account for the significant morbidity and mortality associated with this procedure. The high participation in this survey suggests that a prospective national audit of PEG outcomes is feasible. 